\title{
Ueber quantitative Bestimmung des Morphins im 0pium;
}

von

\section{Dr. von Perger.}

Anlass zu den folgenden vorläufigen Mittheilungen gaben sehr differirende Resultate, welche bei der Untersuchung zweier Proben käuflichen Opiums auf den Gehalt an Morphin, nach verschiedenen Methoden durchgeführt, erhalten worden waren.

Aeussere Gründe bewegen mich, meine diesbezüglich gemachten Wahrnehmungen schon jetzt zu veröffentlichen, obgleich die Arbeit noch keineswegs als abgeschlossen angesehen werden kann, und es noch zahlreicherer Resultate bedarf, um ein endgültiges Urtheil gewinnen zu können. Unter den zahlreichen Methoden, welche zur quantitativen Bestimmang des Morphins im Opium in Vorschlag gebracht wurden, ist die von Herrn E. Merk angegebene, welche auch von den Herren Baur und Finckh empfohlen wurde, eine der besten. In dem grossen Etablissement des Herrn E. Merk in Darmstadt wird dieselbe ausschliesslich angewendet, und sie giebt, wie mir Herr E. Merk mitzutheilen die Liebenswürdigkeit hatte, Resultate, welche mit den Ergebnissen der fabrikmässigen Verarbeitung des Opiums im besten Einklange stehen.

Infolge dessen wurde diese Methode von mir stets angewendet.

Gelegentlich der Untersuchung zweier Proben Opiums, welche von meinem früheren Assistenten, Herrn C. Horn o ch nach der genannten Methode durchgeführt wurde, ergaben sich bedeutende Differenzen mit jenen Resultaten, welche nach der Methode der Pharmac. austr. Ed. VI und der von Herrn Dr. Godeffroy verbesserten Hager'schen Methode erhalten wurden.

Diese divergirenden Resultate konnten nicht in der Ungleichheit des Untersuchungsobjectes gelegen sein, und es schien mir nöthig, den Grund dieser Differenzen aufzuklären. 
Dazu war es vor Allem nöthig, in den Besitz der Originalmethoden zu gelanğen, um an der Hand derselben vergleichende Prüfungen vorzunehmen.

Herr Prof. Dr. Godeffroy hatte die Gefälligkeit, mir den Gang seiner Untersuchungsmethode zu detailliren; derselbe ist folgender:

"Man nimmt $10 \mathrm{Grm}$. getrocknetes Opiumpulver und knetet $\theta \mathrm{S}$ in einer Porzellanschale mit ungefähr $25 \mathrm{Ccm}$. heissen Wassers gut durch, giebt dann die Masse auf ein leinenes Collatorium, presst aus, bringt den Rückstand wieder in die Reibschale, knetet wieder mit etwa $25 \mathrm{Ccm}$. heissem Wasser und wiederholt diese Operationen, Kneten und Auspressen so lange, bis das neu hinzugefügte Wasser nicht mehr gefärbt wird. Die collirte Flüssigkeit vermischt man mit einem Brei aus 8-10 Grm. gelöschten Kalkes, kocht 2-3 Mal auf, filtrirt und löst in dem Filtrate so viel Salmiak auf, dass die Flüssigkeit selbst nach längerem Stehen deutlich nach Ammoniak riecht. Nach 12-24 Stunden ist das Morphin herauskrystallisirt, man bringt es auf ein früher gewogenes Filter, wäscht es mit wenig ammoniakhaltigem Wasser, trocknet und wägt wieder."

Die Methode der Pharmacopoea austriaca Ed. VI ist folgende $\left.{ }^{1}\right)$ :

„10 Grm. gepulvertes, getrocknetes Opium werden mit $90 \mathrm{Grm}$. einer Mischung, bestehend aus $140 \mathrm{Grm}$. destillirten Wassers und $40 \mathrm{Grm}$. Salzsäure $(12,2 \%)$ digerirt. Mit dem Rest der verdünnten Salzsäure $(90 \mathrm{Grm}$.) wird gewaschen.

Der nach dem Filtriren bleibende und gewaschene Rückstand wird getrocknet, und sein Gewicht bestimmt. Wenn das Opium gut ist, darf letzteres nicht 4,5 Grm. überschreiten. Der salzsauren Lösung werden $20 \mathrm{Grm}$. gepulvertes Chlornatrium zugemischt und 24 Stunden in der Kälte stehen gelassen. "Der erhaltene Niederschlag wird durch Decantation und Filtriren von der Flüssigkeit getrennt und auf dem Filter mit einer gesättigten Kochsalzlösung gewaschen.

1) Siehe Commentar zur österr. Pharmacopöe, III. Bd. (Text der neuen Pharmacopöe) von Dr. F. C. Schneider u. Dr. Aug. Vogel, Seite 136. 
$\mathrm{Zu}$ den Flüssigkeiten, Filtrat und Waschwasser giebt man Ammoniak zu, bis die Flüssigkeit deutlich danach riecht. Die Lösung bleibt 12 Stunden stehen, bis sich das Morphin in Krystallen abscheidet, man decantirt, mischt den Niederschlag mit möglichst wenig destillirtem Wasser, spült in ein Filter, trocknet in einer Porzellanschale und versetzt mit dem gleichen Gewicht einer Mischung, welche aus gleichen Theilen Essigsäure $(20,4 \%)$ und destillirtem Wasser besteht; man setzt noch die gleiche Wassermenge hinzu, filtrirt und wäscht das Filter mit ca. $50 \mathrm{Ccm}$. Wasser. Die Flüssigkeiten dürfen das Gewicht von 70-80 Grm. nicht überschreiten. Dann wird zu dem Filtrat Ammoniak in geringem Ueberschuss gegeben und 12 Stunden stehen gelassen; man sammelt auf gewogenem Filter, wäscht mit $20 \mathrm{Ccm}$. Wasser und trocknet. Das Minimum muss 1 Grm. betragen." Infolge meines Ersuchens theilte mir Herr E. Merk seine Methode mit, sie lautet:

„15 Grm. zerschnittenes Opium werden mit $100 \mathrm{Grm}$. Spiritus von $45 \%$ Gehalt ausgekocht, der Auszug wird durch Filtration vom Rückstand getrennt und der Rückstand nochmals mit $100 \mathrm{Grm}$. Spiritus von derselben Stärke behandelt; die vereinigten Auszüge werden mit $8 \mathrm{Grm}$. kryst. Soda versetzt und ohne umzurühren eingedampft. Der erhaltene Abdampfrückstand wird mit $60 \mathrm{Grm}$. kaltem Wasser aufgeweicht, in einem Cylinderglase decantirt, die klare Flüssigkeit abgegossen und das Ungelöste nochmals mit $30 \mathrm{Grm}$. kalten Wassers abgewaschen, dann in derselben Weise mit $45 \mathrm{Grm}$. Weingeist (von $90 \%$ Gehalt) behandelt und schliesslich auf ein Filter gebracht. Die auf dem Filter zurückbleibende, krystallinische Masse trocknet man zwischen Filterpapier ab, löst in einer Mischung von $15 \mathrm{Grm}$. Essigsäure (bestehend aus $1 \mathrm{Thl}$. Essigsäure von der Dichte 1,06 und 8 Thln. Wasser) und 15 Grm. destill. Wasser, filtrirt durch dasselbe Filter, auf wekchem der Rückstand gesammelt wurde, fällt das Filtrat mit Ammoniak und sammelt nach 12 stündigem Stehen den Niederschlag auf einem tarirten Filter, trocknet und wiegt."

Von dem Grundsatze ausgehend, dass eine analytische 
Methode nur dann sichere Resultate geben kann, wenn durch unausgesetzte Prüfung, durch qualitative, empfindliche Reactionen die quantitativ zu bestimmende Verbindung bei den analytischen Operationen stets verfolgt wird, dass das erhaltene Produkt, welches gewogen wird, die möglichste Reinheit besitzen muss, stellte ich die nachstehende Methode für die Morphinbestimmung in Opium als Vergleichungsmittel zusammen:

10 bis $20 \mathrm{Grm}$. des zu untersuchenden Opiums werden in möglichster Vertheilung mit 15 bis 30 Grm. Aetzbaryt ${ }^{1}$ ) und ca. 150-200 Ccm. Wasser ganz kurze Zeit gekocht; die Lösung wird durch Decantation und Filtriren von dem Rückstande getrennt, und letzterer mit kleinen Mengen heissen Wassers noch einige Male gekocht, bis eine Probe der Lösung, verdunstet, mit Molybdänsäure-Schwefelsäure keine Morphinreaction mehr zeigt. Bei dieser Operation ist ein $\mathrm{zu}$ oft wiederholtes Auskochen zu vermeiden, und betragen die Filtrate in der Regel nicht mehr als etwa 400-500 Ccm. Volumen. In diese Lösung, welche alles Morphin enthält, leitet man einen Strom gewaschener Kohlensäure ein, bis die Flïssigkeit mit dem Gase übersättigt ist, und dampft nun die ganze Masse möglichst rasch in einer Schale zur Trockne auf dem Wasserbade ein.

Den erhaltenen Rückstand befeuchtet man mit absolutem Alkohol, löst ihn mittelst eines scharfen Spatels von der Schale los, bringt ihn in einen Glaskolben (nach Erlenmeyer) und extrahirt nun mit kochendem absoluten Alkohol so lange, bis eine Probe desselben, auf einem Uhrglasè verdunstet, keine deutliche Morphinreaction mit Molybdän-Schwefelsäure mehr zeigt. Dazu genügen in der Regel ca. $300-400$ Ccm. Die klaren alkoholischen Filtrate werden in einem kleinen Kolben (Erlenmeyer) durch Destillation vom Alkohol befreit. ${ }^{2}$ ) Ist durch wiederholtes Nach-

1) Die Anwendung so bedeutender Mengen von Aetzbaryt ist unbedingt nöthig und wird durch die nenen Angaben von D. B. Dott (Aus Chem. and Drugg.d.Deutsch-Amerik. Apoth.-Ztg. 4, 571) begründet.

2) Die Extraction gelingt um so rascher, je feiner zertheilt der Eindampfrückstand ist, und diese Zertheilung lässt sich in der Schale 
giessen von Alkohol und Destillation das Volum der Lösung ein kleines geworden, so wird der Rest des vorhandenen Alkohols durch Einstellen des Kolbens in ein kachendes Wasserbad vollständig verdamptt.

Der im Kolben verbleibende Rückstand ist von gelbbrauner Farbe, harzartig, klebrig; man giesst nun auf denselben ca. $15 \mathrm{Ccm}$. Wasser, dem man etwas Ammoniak zngefügt hat, und lässt längere Zeit stehen. Die dunkle, harzartige Masse färbt sich lichtbraun und wird fest durch die Aufnahme von Wasser. Die Umwandiung wird durch Rühren und Reiben mit einem, mit Kautschuk versehenen Glasstab befördert. Ist die Masse derart verändert, so wird durch ein kleines, gewogenes Filter decantirt und der fein vertheilte Niederschlag durch wiederholtes Zurückgiessen des Filtrates auf das Filter gebracht; schliesslich wäscht man mit wenig ammoniakalischem Wasser nach. Auch hier sind grössere Flüssigkeitsmengen möglichst zu vermeiden. Das Filter wird bei $40^{\circ}$ getrocknet und in einen Glastrichter gebracht, welcher unten geschlossen werden kann. Man übergiesst das Filter mit reinem, alkoholfreiem Chloroform und lässt letzteres mit dem Filterinhalt längere Zeit in Berührung, dann öffnet man den Hahn, lässt das Chloror form ablaufen, giesst eine neue Menge desselben auf und wiederholt die Operation so lange, bis eine Probe des nur wenig mehr gefärbten Extractionsmittels, auf einem Uhrglase verdunstet, der bleibende Rückstand in verdünnter Salzsäure gelöst und die Lösung mit Aetznatronlösung versetzt, nur mehr eine spurenweise Trübung zeigt (3-5 Cem. der Chloroformlösung genügen zu dieser Probe).

Das Filter wird getrocknet und gewogen; das erhaltene Produkt ist rohes Morphin. Dasselbe muss lichtbraun bis strohgelb gefärbt sein; es enthält kein Narcotin und ist in manchen Fällen ${ }^{1}$ ) schon so rein, dass es, mit sehr wenig

mit Hilfe eines Pistilles leicht bewerkstelligen. Die Destillation des Alkohols erfolgt am besten durch directes Eihitzen des Kolbens auf einem Drahtnetze.

1) Die Reinheit hängt von der Qualität des Opiums und von der exacten Durchführung der Methode ab. 
Salzsäure tubergossen, sich mit gelber Farbe löst und beim Schütteln die Lösung durch Bildung des salzsauren Salzes sofort krystallinisch erstarrt; ein allmähliges Krystallisiren aus Alkohol liefert reine Morphinkrystalle; sämmtliche Reactionen des Morphins werden erhalten.

Aus einem ägyptischen Opium auf die beschriebene Weise in grösserer Menge erzeugtes Rohmorphin sandte ich Herrn E. Merk in Darmstadt zur Begutachtung ein, und derselbe erklärte es (bis auf Spuren von Narcotin) als rein.

Nicht jedes Opium liefert ein so reines "Rohmorphin“ und ist demnach in jedem Falle eine Reinigung desselben durchzuführen; meistens enthält das derart gewonnene Produkt noch: 1) in verdünnter Essigsäure unlösliche Körper, und 2) Verbindungen, welche sich in verdünnter Essigsäure lösen, durch Ammoniak und durch gelbes Blutlaugensalz gefällt werden.

Ich fand, dass die Menge dieser Verunreinigungen stets eine geringe war, und die Differenz in den Gewichten des Roh- und Reinmorphins hauptsächlich durch den bei der Reinigung erfolgenden Verlust an Morphin bedingt ist, welcher in der, auch bei den anderen Methoden in Anwendung kommenden Krystallisation aus Ammoniak zu suchen ist.

Die Reinigung des Rohmorphins erfolgt, indem man das Filter mit dem Rohmorphin mit möglichst wenig, sehr verdünnter Essigsäure übergiesst. (Bei einem Gewicht des Rohmorphins von 1,0-1,5 Grm. genügt eine Mischung von $1,5-2 \mathrm{Ccm}$. Essigsäure $(20 \%)$ und $20-25 \mathrm{Ccm}$. Wasser.) Um die Lösung des Morphins als Acetat zu fördern, kann man die verdünnte Essigsäure auch erwärmt $\left(30^{\circ}-50^{\circ}\right)$ anwenden. Man wiederholt das Aufgiessen mit dem Filtrate mehrmals, um alles Morphin zu lösen, wäscht mit $5 \mathrm{Ccm}$. Wasser nach, und versetzt nun das gelbbraune Filtrat mit einigen Tropfen von Ferrocyankaliumlösung, filtrirt wieder durch dasselbe Filter und wäscht mit ca. 20-25 Ccm. Wasser nach. ${ }^{{ }^{2} \text { ) }}$

1) Je grösser die in Anwendung kommenden Wassermengen sind, desto grösser ist der Verlust an Morphin. Diesen Verlust durch eine Correctur zu compensiren, sind Versuche im Gange. 
Das gesammte Volumen darf $50-60 \mathrm{Ccm}$. nicht übersteigen. ${ }^{2}$ )

Die erhaltene Lösung wird mit Ammoniak alkalisch gemacht und bleibt 24 Stunden sich selbst überlassen; das krystallisirte Morphin wird auf einem getrockneten gewogenen Filter gesammelt, mit ammoniakalischem Wasser gewaschen, bei $102^{\circ}$ getrocknet und gewogen.

Das derart gewomnene Produkt besitzt eine weisse bis strohgelbe Farbe; es löst sich in verdünnter Natronlauge leicht und vollständig auf, mit wenig Salzsäure übergossen erstarrt die Lösung rasch durch die krystallinische Ausscheidung von reinem salzsauren Morphin, aus Alkohol krystallisirt, erhält man die für das reine Morphin charakteristischen Krystalle; mit gelbem Blutlaugensalz entsteht in saurer Lösung keine Trübung; alle für das Morphin charakteristischen Reactionen werden in einer für die Reinheit des Produktes sprechender Form erhalten. ${ }^{2}$ )

Die gewogene Menge des Produktes auf Procente umgerechnet giebt die Minimalmenge des im untersuchten Opium enthaltenen Morphins an, da durch die Reinigung stets ein Verlust an Morphin erfolgt.

Im Nachstehenden gebe ich einige nach den obengenannten Methoden bei Untersuchung verschiedener Sorten Opium gewonnene Resultate an. Die diesbezüglichen Versuche wurden von mir unter Mithilfe meines Herrn Collegen J. V. Janovsky, der sich mehrfach der Mühe unterzog, die von mir zusammengestellte Methode zu prüfen, im Vereine mit dem Herrn Collegen R. Kämpf, den Herren As-

1) Sollten die Flüssigkeitsmengen nach der Extraction mit Essigsäure grössere sein, als die angegebenen, so kann auf dem Wasserbade eingeengt werden, jedoch muss dies vor dem Zusatz des Blutlaugensalzes geschehen.

2) Bei allen bisher untersuchten Opiumproben gelang es jedesmal ein so reines Produkt zu gewinnen, wenn die angegebenen Bedingungen eingehalten wurden, das Auskochen mit Barytwasser nicht zu weit geführt und die Menge der zum Lösen nöthigen Essigsäure nicht unnöthig vermehrt wurde, wodurch eine spurenweise Lösung der durch Ferrocyankalium fällbaren Körper eintritt. 
sistenten C. Hornoch und R. Spängler durchgeführt und spreche ich den genannten Herren für die freundliche Unterstützung meinen verbindlichsten Dank aus.

I. a. Opium mit einem ursprünglichen Wassergehalt von 12,23\% ergab, nach der Methode der Pharm. austriac. Ed. VI untersucht, aus 10,23 Grm. trocknen Opiums 0,427 Grm. eines Produktes, welches sich als sehr unreines Morphin erkennen liess, 4,17\% ${ }^{1}$ ) (auf Trockenrückstand bezogen).

b. Eine Probe desselben Opiums gab nach der Methode von Hager-Godeffroy: aus 10,425 Grm. trocknen Opiums 0,17 Grm. Morphin = 1,63\% $\% .^{2}$ ) Das erhaltene Produkt war in Salzsäure mit brauner Farbe löslich, gab aber alle charakteristischen Morphinreactionen (H.).

c. Eine Probe desselben Opiums ergab nach der von mir zusammengestellten Methode, welche ich der Kürze halber mit IV bezeichne, aus 10,35 Grm. trocknen Opiums 1,067 Grm. Rohmorphin (aschenfrei, nach Reinigung mit Chloroform). Das gewonnene Produkt war sehr rein und enthielt nur Spuren in Essigsäure unlöslicher Körper.

d. Eine Probe Opium, derselben Sendung entstammend, gab nach der Methode IV aus 10,74 Grm. trocknen Opiums 1,095 Grm. Rohmorphin, aus welchem 0,952 Grm. krystall. Morphin erhalten wurden, somit 8,86\%. (Der Wassergehalt des ungetrockneten Opiums betrug 10,35\% $\%^{\circ}$ (H.)

e. Opium gleicher Art gab bei der Untersuchung, nach Methode IV, bei einem Wassergehalte von $8 \%$ : aus 12,07 Grm. trocknen Opiums 1,46 Grm. Rohmorphin, entsprechend $1,15 \mathrm{Grm}$. Reinmorphin, somit $9,52 \%$ oder $8,74 \%$ Morphin vom nativen Opium. (H.)

II. Herr E. Merk hatte die Liebenswürdigkeit, mir ägyptisches Opium zur Disposition zu stellen, welches nach seiner Angabe 4,5\% Morphin (im nativen Opium) enthielt. Der Wassergehalt betrug $24,77 \%$.

1) S. Beilstein, Handb. d. organ. Chem. Bd. 2, S. 1944. (Reactionen des Morphins.)

2) Diese Resultate sind mehrfach bestätigt worden in Schwankungen von $2-4 \%$. 
Diese Opiumprobe wurde vollständig durchgeknetet und eine nochmalige Wasserbestimmung mit der Durchschnittsprobe gemacht; es wurden $22,26 \%$ Wasser gefunden.

a. 12,7 Grm. dieser Probe = 9,873 Grm. trocknen Opiums gaben nach der Methode von Hager-Godeffroy 0,048 Grm. $=0,486 \%$ Morphin (auf trocknes Opium).

b. 12,7 Grm. dieser Probe gaben in gleicher Weise $0,046 \mathrm{Grm} .=0,476 \%$ (auf trocknes Opium). (H.)

c. 9,466 Grm. trocknen Opiums $=12,581$ nativen Opiums gaben, in gleicher Weise behandelt, 0,053 Grm. Morphin = $0,56 \%$ (H.).

In allen drei Fällen konnte mit Methode $I V$ in den Rückständen und den Filtraten noch Morphin quantitativ bestimmt werden.

d. 9,685 Grm. trocknen Opiums $=12,81 \mathrm{Grm}$. der nativen Probe ergaben nach der Methode der Pharm. austr. 2,04\% unreines Produkt.

e. 15 Grm. der gekneteten Probe nach der Methode E. Merk's untersucht, gaben 0,778 Grm. narcotinhaltigen Morphins, welches, mit Chloroform gewaschen, 0,7065 Grm. reinen Morphins gab, somit 4,7\% in der ungetrockneten Probe. Das Produkt war sehr rein.

f. $15 \mathrm{Grm}$. desselben Opiums (11,66 Grm. trocken) gaben nach der Methode E. Merk's 0,731 Grm. $=4,8 \%$ noch narcotinhaltigen Morphins, welches, mit Chloroform gereinigt, $0,697 \mathrm{Grm}$. Reinmorphin ergab $=4,64 \%$ im ungetrockneten Opium. (H.)

g. 10,85 Grm. desselben Opiums gaben nach Methode IV 0,8775 Grm. Rohmorphin, woraus 0,72 Grm. Reinmorphin erhalten wurden, somit 6,63\% auf natives Opium berechnet. (Jan.)

h. $12 \mathrm{Grm}$. nasses Opium nach der Methode IV verarbeitet (9,329 Grm. trocken) gaben 0,99 Grm. Rohmorphin $=0,828 \mathrm{Grm}$. Reinmorphin $=6,9 \%$ auf natives Opium berechnet. (H.)

i. 12,7 Grm. desselben Opiums, nach Methode IV verarbeitet, gaben $0,9788 \mathrm{Grm}$. Rohmorphin $=0,764 \mathrm{Grm}$. Reinmorphin $=6,01 \%$ auf die ursprüngliche Probe berechnet. 
Nach den Analysen g, h, i stellt sich der mittlere Gehalt an Morphin auf 6,51\% im ursprünglichen, oder $8,37 \%$ im bei $105^{\circ}$ getrockneten Opium.

III. a. Eine Probe Opium von dunklem Aussehen, mit einem Wassergehalt von nur $2,1 \%$, wurde vollständig getrocknet und nach der Methode Hager-Godeffroy untersucht. Es wurden $10 \mathrm{Grm}$. viermal ausgeknetet und die Filtrate der Kalkauskochung mit Salmiak versetzt, das Volumen der Lösung betrug $200 \mathrm{Ccm}$. Nach 24stündigem Stehen wurden erhalten: 0,281 Grm. Morphin; nach 24 Stdn. wurde wieder filtrirt, erhalten wurden $0,258 \mathrm{Grm}$. unreinen Morphins $=5,39 \%$ Morphin; dasselbe stellte ganz kleine, kugelförmige, dunkle, krystallinische Massen dar, welche sich in Salzsäure mit rothbrauner Farbe lösten; mit Aetznatron gab diese Lösung noch eine schwache Fällung. Aus den Rückständen und den Filtraten wurden nach der Methode IV noch gewonnen 0,55 Grm. Rohmorphin $=0,366$ Grm. Reinmorphin, somit $3,66 \%$. Der Gesammtgehalt war auf diese Weise bis auf $9 \%$ gebracht worden.

b. 12,253 Grm. trocknen Opiums derselben Probe wie IIIa entstammend, gab nach 24 Stdn. 0,524 Grm. Morphin von gleichem Aussehen, somit 4,1\%; nach $36 \mathrm{Stdn}$. waren noch 0,18 Grm. auskrystallisirt, im Ganzen wurden somit gewonnen $0,704 \mathrm{Grm} .=5,745 \%$ Morphin im trocknen Opium. (H.)

c. 12,5 Grm. derselben Probe $=12,253$ Grm. trocken, gaben nach Methode IV 1,422 Grm. Rohmorphin = 1,115 Grm. Reinmorphin, somit $9,1 \%$.

IV. a. Ein Opiumbrod durch Hrn. Droguisten H. Siegmund von Gehe u. Co. bezogen, gab bei einem Trockengehalt von $80,52 \%$, in 9,968 Grm. einer getrockneten Probe, nach der Methode der Pharmacop. austr. verarbeitet, 0,253 Grm. eines unreinen Produktes.

b. Zwei in gleicher Art durchgeführte Proben mit je $10 \mathrm{Grm}$. des getrockneten Opiums gaben ganz unreine Produkte, welche nicht als Morphin angesehen werden konnten. (Sp.) 
c. $10 \mathrm{Grm}$. des getrockneten Opiums gaben nach der Methode Hager-Godeffroy in concentrirtester Lösung 1,173 Grm. eines stark narcotinhaltigen Morphins, welches nach dem Waschen mit Chloroform ein sehr reines Produkt lieferte $=0,787 \mathrm{Grm}$.

In diesem Falle wurden, da der Wassergehalt 18,92\% betrug, erhalten: Rohmorphin $=11,73 \%$, Reinmorphin $=$ $9,71 \%$ auf trocknes Opium, 9,51\% Rohmorphin $=7,87 \%$ Reinmorphin auf nasses Opium berechnet. (Sp.)

d. 9,046 Grm. des getrockneten Opiums der Durchschnittsprobe gaben 0,8037 Grm. = 8,88\% Rohmorphin (narcotinhaltig) nach der Methode Hager-Godeffroy, welches nach Behandlung mit Chloroform 7,34\% Reinmorphin lieferte $=5,99 \%$ im ursprünglichen, feuchten Opium. (Sp.)

e. 14,908 Grm. Opium nativ (Wassergehalt 18,92\%), nach der Methode E. Merk's verarbeitet, gaben 1,234 Grm. $=8,01 \%$ narcotinhaltiges Morphin, nach der Reinigung mit Chloroform 7,56\% im ungetrockneten Opium. Das gewonnene Produkt war sehr rein. (Sp.)

f. 10,3195 Grm. desselben Opiums (Wassergehalt 19,48\%) gaben, nach Methode IV verarbeitet, 1,445 Grm. Rohmorphin und 0,89 Grm. Reinmorphin, somit 8,64\% Reinmorphin auf natives, oder $10,73 \%$ Morphin auf trocknes Opium.

g. $10 \mathrm{Grm}$. feuchten Opiums wurden nach der Methode IV verarbeitet (Wassergehalt 19,43\%). An Robmorphin wurden gewonnen $12,167 \%$, an Reinmorphin $9,024 \%=11,42 \%$ im trocknen Opium. (Jan.)

h. $10 \mathrm{Grm}$. nativen Opiums wurden wie unter g behandelt, das Rohmorphin betrug $11,07 \%$, das Reinmorphin $8,78 \%$ im nativen Opium, somit $10,89 \%$ auf trocknes Opium umgerechnet. (Jan.)

Die Differenzen in den Resultaten waren bei diesem Opium gegenüber den früher angeführten verhältnissmässig kleine.

V. a. Ein ägyptisches Opium von braunem, unschönem Aussehen, durch Hrn. H. Siegmund von E. Merk in Darmstadt bezogen, gab nach der Methode Hager-Godeffroy 
aus $10 \mathrm{Grm}$. 0,147 Grm. eines Produktes, welches keine Morphinreaction gab. (K.)

b. In einem zweiten Falle wurden $0,78 \%$ eines Körpers gewogen, der kein Morphin war. $(\mathrm{K}$.)

c. $10 \mathrm{Grm}$. trocknen Opiums gaben, nach derselben Methode behandelt, 0,127 Grm. eines Körpers, der keine Morphinreaction zeigte. (Sp.)

d. 15 Grm. dieses Opiums gaben nach der Methode Merk's verarbeitet, 0,3195 Grm. eines unreinen Produktes $(2,13 \%)$, dasselbe löste sich beim Behandeln mit Chloroform zum Theile auf. (Sp.)

e. $15 \mathrm{Grm}$. dieses Opiums gaben, in gleicher Weise behandelt, 0,29 Grm. Rohmorphin =1,93\%, welche an Reinmorphin $0,258 \mathrm{Grm} .=1,72 \%$ gaben. $(\mathrm{K}$.

f. Nach der Methode der Pharmacop. wurden aus 10,119 Grm. getrockneten Opiums $0,3 \%$ eines unreinen Produktes erhalten.

g. $15 \mathrm{Grm}$. desselben Opiums, dessen Wassergehalt 9,61\% betrug, gaben, nach der Methode IV behandelt, 0,903 Grm. Rohmorphin und nach der Reinigung an Reinmorphin $=3,6 \%$ (Sp.)

h. Aus 10,114 Grm. desselben Opiums wurden 0,545 Grm. Rohmorphin und 0,311 Grm. Reinmorphin, somit 3,07\% erhalten (Methode IV).

VI. a. Eine Probe von ächt persischem Opium vorzüglicher Qualität wurde nach der Methode Hager-Godeffr oy untersucht. Aus $10 \mathrm{Grm}$. des getrockneten Opiums (Wassergehalt 16,7\%) wurden erhalten: 0,9073 Grm. unreinen Morphins. Nach der Behandlung mit Chloroform wurden erhalten 0,812 Grm. eines sehr reinen Produktes, somit 8,12\% Morphin im trocknen $=6,76 \%$ im ursprünglichen Opium. (Sp.)

b. 10 Grm. des getrockneten Opiums wurden nach derselben Methode untersucht; erhalten wurden: 1,149 Grm. unreinen Morphins, welches nach Reinigung mit Chloroform 0,277 Grm. verlor (hauptsächlich Narcotin) und dessen Gewicht daher $0,872 \mathrm{Grm}$. betrug. In dem Falle wurden also an Reinmorphin im trocknen Opium $8,72 \%$ gefunden, in der nativen Probe $7,26 \%$ reines Morphin. (K.) 
c. Dasselbe Opium nach der Methode Merk untersucht: in $15 \mathrm{Grm}$. der wasserhaltigen Probe wurden gefunden 1,844 Grm. nicht ganz reinen Morphins, welches nach der Reinigung mit Chloroform 1,696 Grm. wog. Der Reingehalt im nativen Opium betrug somit $11,31 \%$ oder im trocknen Opium $13,57 \%$. (Sp.)

d. Aus 10,5145 Grm. nassen Opiums wurden nach der Methode IV gefunden: an Rohmorphin 1,61 Grm., an Reinmorphin $1,268 \mathrm{Grm}$., somit $12,05 \%$ im nativen, $14,46 \%$ im trocknen Opium.

e. Dasselbe Opium: $15 \mathrm{Grm}$. desselben gaben nach Methode IV 2,3402 Grm. Rohmorphin und 2,053 Grm. Reinmorphin, somit $13,67 \%$ Reinmorphin auf natives Opium oder $16,41 \%$ auf trocknes Opium berechnet. (Sp.)

f. 15,6 Grm. desselben Opiums gaben nach derselben Methode 1,742 Grm. Reinmorphin, somit $11,17 \%$ auf natives oder $13,4 \%$ auf trocknes Opium berechnet. In dem Falle wurde aus verdünnterer Lösung durch Ammoniak gefällt. (Jan.)

g. Nach der Methode der Pharmacopöa gelang es bisher nicht ein nur einigermaassen reines Produkt zu erhalten.

Uebersichtshalber stelle ich hier die erhaltenen Durchschnittswerthe nach den verschiedenen Methoden, bezogen auf getrocknetes Opium, zusammen, wobei die unreinen Produkte mit * bezeichnet sind:

Gehalt an Morphin:

Hager-

Opium. E. Merk. Pharm, austr., Godeffroy. Methode IV.,

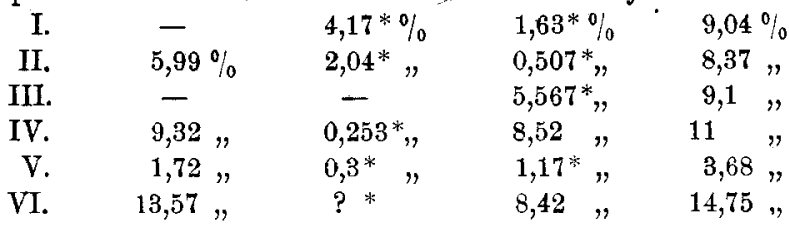

Diese Resultate der Untersuchung, aus welchen ich vorläufig noch keine definitiven Schlussfolgerungen ziehen will, da die Zahl derselben eine noch zu kleine ist, zeigen so überraschende Differenzen, dass bei aller Rücksichtsnahme auf die 
Schwierigkeit, genaue Durchschnittsproben zur Analyse zu bringen, eine Erklärung der Unterschiede der nach den verschiedenen Methoden erhaltenen Produkte sowohl in qualitativer als quantitativer Beziehung, nur in der Art der Methoden und deren subjectiven Durchführung gesucht werden kann.

Bei der Wichtigkeit der Frage ist zu erwarten, dass einige meiner Fachgenossen durch diese vorläufige Mittheilung angeregt werden dürften, diese Sache näher zu studiren. Weitere Mittheilungen behalte ich mir vor.

Reichenberg, Laboratorium für chem. Technologie, im Februar 1884.

\section{Studien über Milch; \\ von}

Heinrich Struve.

\section{Dritte Mittheilung. ${ }^{1}$ )}

Frauenmilch.

In den letzten Jahren wurde ich von verschiedenen Aerzten und in Sonderheit von dem Vorstand der Tifliser geburtshülflichen Anstalt ersucht, Proben von Frauenmilch auf ihre Güte hin zur Auswahl von Ammen zu prüfen. Bei dieser Gelegenheit unterliess ich es nicht, die im Verlauf meiner Arbeiten über Kuhmilch gesammelten Erfahrungen anzuwenden und $\mathrm{zu}$ verwerthen. Freilich war dieses nicht in allen Fällen möglich, da nur zu oft die mir zur Prüfung überschickten Milchproben eben zu den einfachsten Proben hinreichten. In solchen Fällen beschränkte ich mich ausschliesslich auf die Prüfung mit Hülfe des Apparates von F'eser, auf die Ausschüttelung mit Aether und auf die mikroskopische Untersuchung. Lag ein grösseres Volum Milch

1) Vergl. die zweite Mittheilung dies. Journ. [2] 29, 70. 